\title{
过氧硝酸乙酰酯分解反应的速率常数
}

\author{
韦文美郑仁慧田燕何天敬 ${ }^{*}$ 陈东明刘凡镇 \\ (中国科学技术大学化学物理系, 合肥 230026)
}

摘要 在 G3MP2B3 结构优化和能量计算的基础上, 采用 RRKM 理论和疏松过渡态模型重新估算了过氧硝酸 乙酰酯( $\mathrm{PAN})$ 的热分解反应 $\mathrm{PAN} \rightarrow \mathrm{CH}_{3} \mathrm{C}(\mathrm{O}) \mathrm{OO}+\mathrm{NO}_{2}(\mathrm{R} 1)$ 的反应速率常数, 得到与实验值吻合的结果. 用同样的 模型计算了 $\mathrm{PAN} \rightarrow \mathrm{CH}_{3} \mathrm{C}(\mathrm{O}) \mathrm{O}+\mathrm{NO}_{3}(\mathrm{R} 2)$ 的反应速率常数. 结果表明, 在相同的反应条件下, $\mathrm{R} 1$ 是主要的分解通 道, $\mathrm{R} 2$ 是次要通道, $\mathrm{R} 2$ 的反应速率常数比 $\mathrm{R} 1$ 的小两个数量级.

关键词：过氧硝酸乙酰酯，G3MP2B3， RRKM 理论，反应速率常数，疏松过渡态 中图分类号: 0643

\section{Theoretical Studies of the Rate Constants for Peroxyacetyl Nitrate Decomposing to $\mathrm{CH}_{3} \mathrm{C}(\mathrm{O}) \mathrm{O}+\mathrm{NO}_{3}$}

\author{
WEI, Wen-Mei ZHENG, Ren-Hui TIAN, Yan HE, Tian-Jing ${ }^{*} \quad$ CHEN, Dong-Ming LIU, Fan-Chen \\ (Department of Chemical Physics, University of Science and Technology of China, Hefei 230026, P. R. China)
}

\begin{abstract}
The rate constants for the thermal decomposition reaction of peroxyacetyl nitrate (PAN), $\mathrm{PAN} \rightarrow \mathrm{CH}_{3} \mathrm{C}(\mathrm{O}) \mathrm{OO}+$ $\mathrm{NO}_{2}(\mathrm{Rl})$ were reestimated by RRKM theory and loosing transition state model based on the optimized structures and energies at the G3MP2B3 level of theory and the results agree with the experiment values. Then using the same models, the rate constants for another thermal decomposition reaction of PAN, $\mathrm{PAN} \rightarrow \mathrm{CH}_{3}(\mathrm{O}) \mathrm{O}+\mathrm{NO}_{3}(\mathrm{R} 2)$ were studied. In Troe's notation, the RRKM curves are presented by the following expressions for the limiting low- and highpressure rate constants, $k_{0}(1)=8.0 \times 10^{-3} \exp [-11912.8 / T] \mathrm{cm}^{3} \cdot$ molecule $^{-1} \cdot \mathrm{s}^{-1} ; k_{\infty}(1)=2.8 \times 10^{16} \exp [-13531.8 / T] \mathrm{s}^{-1} ; k_{0}(2)=$ $6.1 \times 10^{16} \exp [-13011.9 / T] \mathrm{cm}^{3} \cdot$ molecule $^{-1} \cdot \mathrm{s}^{-1} ; k_{\infty}(2)=4.0 \times 10^{16} \exp [-15463.1 / T] \mathrm{s}^{-1}$. The results of calculation suggested that the reaction $\mathrm{R} 1$ is the main dissociation channel, while $\mathrm{R} 2$ is the minor one and the difference between the rates of the two reactions is about $10^{2}$ at the same conditions.
\end{abstract}

Keywords: Peroxyacetyl nitrate, G3MP2B3, RRKM theory, Rate constant, Loose transition state

过氧硝酸乙酰酯(PAN)是由人造的或生物体系 中的有机化合物氧化降解形成的大气成分 ${ }^{[1-2]}$, 在 1956 年由 Stephens 等人 ${ }^{[3]}$ 首次在洛杉矶烟雾中检测 到. PAN 是光化学烟雾的重要氧化剂成分 ${ }^{[4]}$, 在对流 层中起传送 $\mathrm{NO}_{x}$ 的重要作用 ${ }^{[0]}$, 并且是对流层中发生 反应的含量最丰富的含氮化合物之一 ${ }^{[0-7]}$. 正是因为 在大气化学中的这种中枢的作用, 科学家对 PAN 的全球性分布进行了广泛的测量 ${ }^{[6-7]}$, 而且对它的热
化学 ${ }^{[5,8-24]}$ 和光化学 ${ }^{[25-29)}$ 性质进行了一系列的研究. 研 究表明, PAN 的损失机制在对流层的上部主要是光 分解,在低层大气中 $(<7 \mathrm{~km})$ 主要是热分解.

PAN 中的 $\mathrm{O}-\mathrm{N}$ 键很弱, 在热分解中它很容易 断裂, 因此几乎所有早先的实验研究都表明 PAN的 热分解只有通过 $\mathrm{O}-\mathrm{N}$ 键的断裂 $[9-12,14,16-20,24]$ 来实现,

$$
\mathrm{PAN} \rightarrow \mathrm{CH}_{3} \mathrm{C}(\mathrm{O}) \mathrm{OO}+\mathrm{NO}_{2}
$$

科学家们已经采用各种不同的方法测定了 PAN 的

Received:June 22, 2005; Revised:August 1, 2005. *Correspondent, E-mail:tj16@ustc.edu.cn; Tel:0551-3607409.

国家教育部博士点基金(2002035806)资助项目

C. Editorial office of Acta Physico-Chimica Sinica 
气相热稳定性和分解反应 R1 的反应速率常数, 并 得到它的阿伦尼乌斯公式 ${ }^{[9-14]}$. 在此基础上, Bridier 等人 ${ }^{[14]}$ 使用 Troe 等的 RRKM 算法 ${ }^{[30-32]}$ 得到了反应 R1 随温度和压强变化的速率表达式, 结果与实验吻 合得很好.

此外, Miller 等人 ${ }^{[8]}$ 在理论上提出了 PAN 的另 外一个热分解反应通道, 即

$\mathrm{PAN} \rightarrow \mathrm{CH}_{3} \mathrm{C}(\mathrm{O}) \mathrm{O}+\mathrm{NO}_{3}$

我们的理论研究也表明 $\mathrm{R} 2$ 可能是除 $\mathrm{R} 1$ 之外的另 一个 PAN 热分解途径 ${ }^{[33]}$. 最近 Ahsen 等人 ${ }^{[15]}$ 预测, 与 $\mathrm{O}-\mathrm{N}$ 键断裂的反应通道相比, $\mathrm{O}-\mathrm{O}$ 键断裂反应 是一个较弱的反应通道, 并且在光谱上可以证实它 的初级反应产物再次分解所得的二级反应产物. 虽 然理论和实验上都已经证明了 R2 是 PAN 热分解 仅次于 R1 重要性的反应通道, Miller 等人 ${ }^{[8]}$ 也通过 定性的量化计算指出 $R 1$ 和 R2 的能垒, 科学家们也 已经采用各种不同的方法在理论和实验上给出了 R1 随温度和压强变化的反应速率常数并得到它的 阿伦尼乌斯公式 ${ }^{[9-14]}$, 但是没有任何理论或者实验进 行 $\mathrm{R} 2$ 的动力学研究, 给出它的反应速率常数.

我们基于 G3MP2B3 的理论计算, 根据 Troe 的 RRKM 计算方法, 重新估算了 $\mathrm{Rl}$ 的反应速率常数, 得到与实验值一致的 RRKM 曲线, 并计算了 $\mathrm{R} 2$ 的 反应速率常数, 得到了它的低压和高压极限的反应 速率常数的表达式, 最后给出了这两个反应的相对 产率.

\section{1 计算方法}

研究表明, 以 Becke 的三参数交换泛函为基础 的杂化密度泛函理论(hybrid DFT)计算所得 PAN 分 子的几何结构和红外振动频率, 与实验结果吻合较 好 ${ }^{[8,15}$. 采用 B3LYP 方法, 即 Becke 的三参数非局域 交换泛函 ${ }^{[34]}$ 与 Lee 等 ${ }^{[35}$ 的非局域相关泛函的 DFT方 法, 以 6-31G $(d)$ 为基组优化了 $\mathrm{CH}_{3} \mathrm{C}(\mathrm{O}) \mathrm{OONO}_{2}$ 及其分 解产物的几何构型, 并在同等水平上进行频率计算. 最后, 用 G3MP2B3 方法 ${ }^{[36}$ 计算了 $\mathrm{CH}_{3} \mathrm{C}(\mathrm{O}) \mathrm{OONO}_{2}$ 分 解反应的势能面. 全部计算工作用 Gaussian 03 程 序包 ${ }^{[37]}$ 在 Pentium IV 计算机上完成.

\section{RRKM 计算}

使用 Troe 的 RRKM 方法 ${ }^{[30-32]}$ 对 PAN 热分解反 应R1 的动力学数据进行了重新估算, 并对 PAN 热分 解反应R2 的速率进行计算. Troe 的 RRKM 算法的
解析式 ${ }^{[30]}$ 为

$$
\begin{aligned}
\lg k(T, c)= & \lg \left\{k_{0}(T) c /\left\{1+k_{0}(T) c / k_{\infty}(T)\right\}\right\}+ \\
& \lg F_{d} d\left\{1+\left\{\lg \left(k_{0}(T) c / k_{\infty}\right) N_{c}\right\}^{2}\right\}
\end{aligned}
$$

其中 $k_{0}(T)$ 和 $k_{\infty}(T)$ 分别是低压极限和高压极限反应 速率常数. $c$ 是反应物浓度, $N_{\mathrm{c}}=0.75-1.27 \lg \left(F_{\mathrm{c}}\right)$, 其中 $F_{\mathrm{c}}$ 是压降曲线增宽系数, 它由微正则分解反应速率 常数的能量依赖和弱碰撞效应引起. 低压极限反应 速率常数表达式为

$$
\begin{aligned}
k_{0}(T)= & c Z_{\mathrm{LJ}} \beta_{\mathrm{c}} \rho_{\text {vibh },}\left(E_{0}\right) k_{\mathrm{B}} T / Q_{\text {vib }} \exp \left[\left(-E_{0}\right) /\right. \\
& \left.k_{\mathrm{B}} T\right] F_{\mathrm{E}} F_{\text {anh }} F_{\text {rot }} F_{\text {rot int }}
\end{aligned}
$$

其中 $Z_{\mathrm{LJ}}$ 是 Lennard-Jones 碰撞频率, $\beta_{\mathrm{c}}$ 是弱碰撞系 数, $\rho_{\mathrm{vibh}}\left(E_{0}\right)$ 是阈能 $E_{0}$ 时的振动态密度, $k_{\mathrm{B}}$ 是玻尔兹 曼常数, $Q_{\text {vib }}$ 是振动配分函数, $F_{\mathrm{E}}$ 是态密度能量依赖 的修正因子, $F_{\text {anh }}$ 表示振动的非谐性修正, $F_{\text {rot }}$ 表示转 动效应的修正因子, $F_{\text {rotint }}$ 表示内转动效应的修正因 子. 高压极限反应速率常数表达式为

$$
k_{\infty}(T)=\left(k_{\mathrm{B}} T / h\right)\left(Q^{\neq} / Q\right) \exp \left(-E_{0} / k_{\mathrm{B}} T\right)
$$

其中 $Q^{\neq} 、 Q$ 分别是过渡态和反应物的配分函数. 参 考文献[14]和[30]已给出 $k(T, c), k_{0}$ 和 $k_{\infty}$ 公式中各项 的含义及计算方法. 具体如下:

（1）假设碰撞是在 Lennard-Jones 势下发生的, 采用 Stiel 和 Thodos 的方法 ${ }^{[30}$ 和相应的临界常数 ${ }^{[3940]}$ 来确定其参数. 对于 PAN, 通过以上参考文献算得 Lennard-Jones 参数 ${ }^{[14]}$ 是 $\sigma_{\mathrm{L}}=0.57 \mathrm{~nm},\left(\varepsilon / k_{\mathrm{B}}\right)_{\mathrm{L}}=410 \mathrm{~K}$, 其中 $\sigma_{\mathrm{L}}$ 是距离参数, $\varepsilon$ 是能量参数;

(2) 因为 PAN 分解反应 R1 和 R2 均没有明显 的能垒 ${ }^{[33]}$, 都属于疏松过渡态类型, 因此, 它们的反 应临界能量取为产物和反应物在 $0 \mathrm{~K}$ 时的焓变, G3MP2B3 结果表明, 分解反应 R1 的临界能量 $\left(E_{0}\right)_{1}=$ $118.8 \mathrm{~kJ} \cdot \mathrm{mol}^{-1}$ (即 $\mathrm{O}-\mathrm{N}$ bond dissociation energy, 以 下简称 $\mathrm{BDE}$ ), $\mathrm{R} 2$ 的 $\left(E_{0}\right)_{2}=132.6 \mathrm{~kJ} \cdot \mathrm{mol}^{-1}$ (即 $\mathrm{O}-\mathrm{O}$ $\mathrm{BDE})$;

（3）假设疏松过渡态位于有效势能面上的最大 点, 它的结构在本质上和反应分子相同. 在形成过渡 态时, 对于反应 $\mathrm{R} 1$, 沿 $\mathrm{O}-\mathrm{N}$ 键伸缩方向为反应坐 标; 对于反应 $\mathrm{R} 2$, 沿 $\mathrm{O}-\mathrm{O}$ 键伸缩方向为反应坐标. 采用 Forst 提到的确定反应类型 1 过渡态的方法 ${ }^{[41]}$, 可以得到 $\mathrm{Rl}$ 的过渡态大概位于 $\mathrm{O}-\mathrm{N}$ 键伸长到 $0.52 \mathrm{~nm}$ 处, $\mathrm{R} 2$ 的过渡态大概位于 $\mathrm{O}-\mathrm{O}$ 键伸长到 $0.50 \mathrm{~nm}$ 处;

(4) 表 1 给出基于 B3LYP/6-31G $(d)$ 计算的 PAN 的振动频率和转动惯量. 由于过渡态的结构疏松, 因 此将 PAN 的代表二面角扭曲振动的四个最低频率 
振子(见表 1 )在过渡态时看成自由转子. 采用折合转 动惯量的方法 ${ }^{[2]} I_{\mathrm{r}}=I_{\mathrm{a}} I_{\mathrm{b}} /\left(I_{\mathrm{a}}+I_{\mathrm{b}}\right)$, 即自由转子的惯量 $I_{\mathrm{r}}$ 为绕自由转动轴两部分转动惯量 $I_{\mathrm{a}}$ 和 $I_{\mathrm{b}}$ 的折合值. 对于 R1 反应的过渡态, 四个自由转子计算得到的 折合转动惯量分别为 $1.95 \times 10^{-38} 、 8.39 \times 10^{-39} 、 5.30 \times 10^{-40}$ 和 $5.83 \times 10^{-39} \mathrm{~g} \cdot \mathrm{cm}^{2}$; $\mathrm{R} 2$ 的分别为 $1.13 \times 10^{-38} 、 8.40 \times$ $10^{-39} 、 5.26 \times 10^{-40}$ 和 $6.27 \times 10^{-39} \mathrm{~g} \cdot \mathrm{cm}^{2}$;

(5) 通过因子 $B$ 来表示过渡态振动频率相对反 应物的降低. 计算最初是在 $T=313.6 \mathrm{~K}$ 时进行的, 这 也是 PAN 分解反应的实验温度范围内一个最普通 的温度, 此时取 $B=1$, 弱碰撞系数 $\beta_{\mathrm{c}}=0.32$ (此时由 Troe, Gilbert 等 ${ }^{[31-32]}$ 的方法得到的 $F_{\mathrm{c}}$ 值为 0.30 ) 能得 到很好地表示分解反应的压降曲线. 如果进一步把 $B$ 值调整到 0.92 , 计算值将会更好地与实验值吻合 (见图 1). 然后在不改变任何参数的情况下把计算的 温度范围(296.9 326.3 K) 展开, 图 1 中给出了理论 拟合的温度分别为 $296.9 、 299.5 、 302.6 、 306.4 、 313.6$ 、 320.9 和 $326.3 \mathrm{~K}$ 下 $\mathrm{PAN}$ 分解为 $\mathrm{PA}+\mathrm{NO}_{2}$ 的曲线, 并画出了相应温度下的实验值, 两者吻合很好. 这表 明 Troe 的 RRKM 计算方法具有 PAN 的动力学性 质的预见性能力 ${ }^{[14]}$ : 用一个温度下校准其压降曲线

\section{表 1 G3MP2B3 理论计算的过氧硝酸乙酰酯的振动频率} 和内转动惯量

Table 1 Vibrational frequencies $\left(f_{\mathrm{v}}\right)$ and moments of inertia $(J)$ of peroxyacetyl nitrate at the level of G3MP2B3

\begin{tabular}{|c|c|c|}
\hline \multicolumn{3}{|c|}{ PAN } \\
\hline \multirow[t]{14}{*}{$f_{\mathrm{v}} / \mathrm{cm}^{-1}$} & 44.3 torsion & 978.7 rock $\mathrm{CH}_{3}$ \\
\hline & 80.1 torsion & 1006.4 rock $\mathrm{CH}_{3}$ \\
\hline & 94.8 torsion & 1073.6 stretch $\mathrm{CO}$ \\
\hline & 97.0 torsion & 1191.0 stretch $\mathrm{O}-\mathrm{O}$ \\
\hline & 314.3 & 1370.4 stretch $\mathrm{NO}_{2}$ \\
\hline & 330.7 & 1420.8 deform $\mathrm{CH}_{3}$ \\
\hline & 370.9 & 1497.5 deform $\mathrm{CH}_{3}$ \\
\hline & 494.2 & 1500.1 deform $\mathrm{CH}_{3}$ \\
\hline & 578.2 & 1841.3 stretch $\mathrm{NO}_{2}$ \\
\hline & 611.9 & 1908.3 stretch $\mathrm{C}=\mathrm{O}$ \\
\hline & 720.3 deform $\mathrm{NO}_{2}$ & 3077.1 stretch $\mathrm{CH}_{3}$ \\
\hline & 726.4 deform $\mathrm{NO}_{2}$ & 3145.1 stretch $\mathrm{CH}_{3}$ \\
\hline & 807.3 stretch CC & 3187.5 stretch $\mathrm{CH}_{3}$ \\
\hline & 837.5 stretch NO & \\
\hline \multirow[t]{3}{*}{$J /\left(\mathrm{g} \cdot \mathrm{cm}^{-2}\right)$} & $I_{\mathrm{A}}$ & $167.6 \times 10^{-40}$ \\
\hline & $I_{\mathrm{B}}$ & $651.1 \times 10^{-40}$ \\
\hline & $I_{\mathrm{C}}$ & $654.8 \times 10^{-40}$ \\
\hline
\end{tabular}

$I_{\mathrm{A}}, I_{\mathrm{B}}$ and $I_{\mathrm{C}}$ are the main moments of inertia relative to the main axises of PAN.
的程序, 可以估计 PAN 分解反应的整个实验的动力 学行为. 因此, 对于 $\mathrm{R} 2$ 分解反应, 采用与 $\mathrm{R} 1$ 反应相 同的弱碰撞系数和过渡态振动频率降低因子来估算 $\mathrm{R} 2$ 的分解反应速率常数.

\section{3 结果与讨论}

根据 Troe 的 RRKM 计算方法和以上给定的参 数,采用非线性最小二乘法得到了 $\mathrm{R} 1$ 和 $\mathrm{R} 2$ 的低压 和高压极限的反应速率常数的表达式. 在此基础上, 研究了这两个反应在一定压强和一定温度下的分解 反应速率常数.

\section{1 分解反应 $\mathrm{R} 1$}

对于分解反应 Rl, Bridier 等人 ${ }^{[14]}$ 在 MNDO/UHF 结构优化和频率计算的基础上已经用 Troe 的RRKM 方法 ${ }^{[30-32]}$ 进行了理论拟合, 我们在 B3LYP/6-31G $(d)$ 计算的基础上在实验的浓度范围内重新拟合得到 $\mathrm{R} 1$ 低压和高压极限的反应速率常数表达式分别为:

$$
\begin{aligned}
& k_{0}(1)=8.0 \times 10^{-3} \exp [-11912.8 / T] \\
& k_{\infty}(1)=2.8 \times 10^{16} \exp [-13531.8 / T]
\end{aligned}
$$

表 2 中列出了计算所得 $\mathrm{R} 1$ 高压极限的反应速率常 数和阿仑尼乌斯参数及相应的实验值.

从表 2 中看出, Bridier 等人 ${ }^{[14]}$ 的高压极限反应 速率常数的指前因子对数值是 16.6 , 阿仑尼乌斯活 化能是 $(113.4 \pm 2.9) \mathrm{kJ} \cdot \mathrm{mol}^{-1}$, 我们拟合的结果分别是 16.4 和 $112.5 \mathrm{~kJ} \cdot \mathrm{mol}^{-1}$, 两者吻合较好. 从此表还可 以看出, 由于实验方法和条件的不同,对于 R1 反应, 不同人得到的实验值不尽相同,尽管这样,除了 Cox 等人 ${ }^{[12]}$ 的实验外, 其他人 ${ }^{[9-11,13-14]}$ 及我们的计算结果都 表明 R1 反应的指前因子达到了 $10^{16} \mathrm{~s}^{-1}$ 数量级甚至

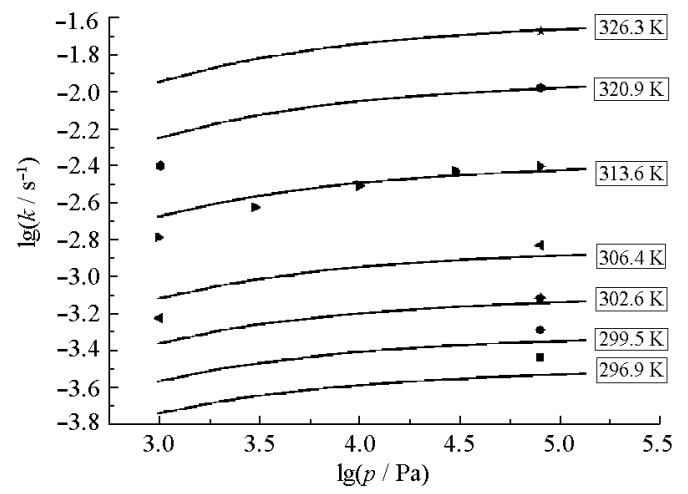

图 1 不同温度下 $\mathrm{R} 1$ 的分解反应速率常数随压强变化曲线

Fig.1 Pressure dependence of total rate constants for the reaction $\mathrm{R} 1$ at different temperatures The points are experimental values reported by Bridier et al. ${ }^{[1]]}$ 
表 2 过氧硝酸乙酰酯热分解反应 $\mathbf{R} 1$ 和 $\mathbf{R} 2$ 的高压极限的反应速率常数与实验值的比较

Table 2 Thermal decomposition rates for R1 and R2 of peroxyacetyl nitrate at the high pressure limit and compare with reported results

\begin{tabular}{llllll}
\hline Reaction & $T / \mathrm{K}$ & $\lg \left(A / \mathrm{s}^{-1}\right)$ & $E_{\mathrm{a}} /\left(\mathrm{kJ} \cdot \mathrm{mol}^{-1}\right)$ & $10^{4} k_{208} / \mathrm{s}^{-1}$ & Ref. \\
\hline R1 & $283 \sim 343$ & 16.4 & 112.5 & 3.5 & This work \\
& $303 \sim 323$ & $17.4 \pm 0.4$ & $119.2 \pm 2.1$ & 3.1 & {$[9]$} \\
& $283 \sim 313$ & $16.4 \pm 0.2$ & $112.5 \pm 2.5$ & 4.2 & {$[10]$} \\
& $288 \sim 298$ & $16.2 \pm 1.6$ & $112.5 \pm 8.8$ & 3.0 & {$[11]$} \\
& $294 \sim 328$ & $14.9 \pm 0.6$ & $104.2 \pm 3.3$ & 4.3 & {$[12]$} \\
& $298 \sim 313$ & $16.3 \pm 0.6$ & $112.5 \pm 3.8$ & 3.7 & {$[13]$} \\
& $296 \sim 314$ & 16.6 & $113.4 \pm 2.92$ & 5.3 & {$[14]$} \\
& $283 \sim 343$ & 16.6 & 128.4 & $8.9 \times 10^{-3}$ & This work \\
\hline
\end{tabular}

更大, 远大于有经典过渡态的一般反应 $\left(10^{13} \mathrm{~s}^{-1}\right)$, 这 表明 PAN 分解成 PA+NO 的过程. 此外, 对比表 2 中 $298 \mathrm{~K}$ 时的 R1 实验分解 速率: $3.1 \times 10^{-4}, 4.2 \times 10^{-4}, 3.0 \times 10^{-4}, 4.3 \times 10^{-4}, 3.7 \times 10^{-4}$ 和 $5.3 \times 10^{-4} \mathrm{~s}^{-1}$, 考虑到实验本身的误差及不确定性, 在 $298 \mathrm{~K}$ 时的 $3.5 \times 10^{-4} \mathrm{~s}^{-1}$ 还是比较好的.

使用以上得到的 $k_{0}(1), k_{\infty}(1)$ 及 $k(T, c)$ 的表达式 分别计算了在压强为 $1.0 \times 10^{3}$ 和 $8.0 \times 10^{4} \mathrm{~Pa}$ 下, 温度 为 $283 \sim 343 \mathrm{~K}$ 时过氧硝酸乙酰酯(PAN)的热分解反 应 R1 的反应速率常数, 并和 Bridier 等 ${ }^{[14]}$ 测得的实 验值进行了比较,结果绘在图 2 中.

从图 2 可以看到温度的变化对 $\mathrm{R} 1$ 的反应速率 常数影响很大, 并且随着温度升高, $\mathrm{R} 1$ 的反应速率 常数逐渐增加, 拟合结果与实验值吻合得很好. 同 样, 为了更清楚地看到 $\mathrm{R} 1$ 反应随反应压强的变化 规律, 我们还绘出了 $298 \mathrm{~K}$ 压强范围为 $1.0 \times 10^{3} \sim$

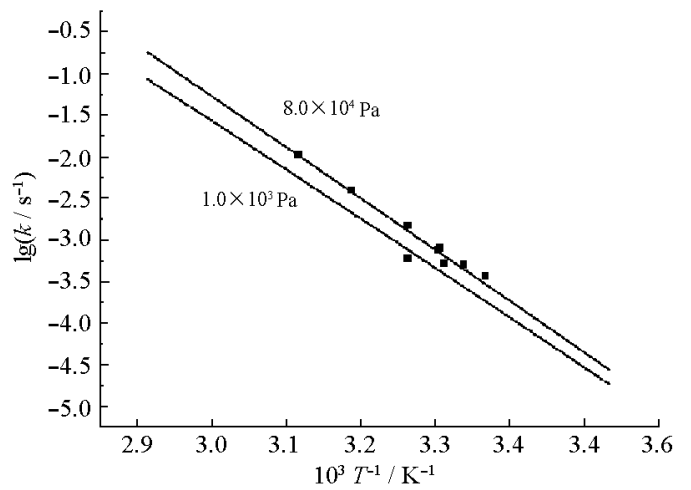

图 2 在 $1.0 \times 10^{3}$ 和 $8.0 \times 10^{4} \mathrm{~Pa}$ 压强下 $\mathrm{PAN}$ 分解反应 $\mathbf{R} 1$ 的阿仑尼乌斯曲线

Fig.2 Arrhenius plots for the decomposition reaction $R 1$ of $P A N$ at the pressure of $1.0 \times 10^{3}$ and $8.0 \times 10^{4} \mathrm{~Pa}$

The points are experimental values reported by Bridier et al.$^{[1]}$
$1.3 \times 10^{5} \mathrm{~Pa}$ 时 $\mathrm{R} 1$ 分解反应速率常数随压强变化曲 线(见图 3).

由图 3 可以看到, 在 $1.0 \times 10^{5} \mathrm{~Pa}$ 的实验条件下, 反应速率常数已经接近最大值, 在 $4.8 \times 10^{3} \mathrm{~Pa}$ 左右 反应速率常数达到最大值的一半. 总之, 对于象 PAN 这样大又没有经典过渡态的分子, 用严格的 RRKM 处理是不现实和不大可能的, 这时, Troe 的 RRKM 计算方法不失为一种有效的处理方法, 它虽 然有一些经验参数, 但因为考虑了很多效应的修正, 能够得到和实验值吻合很好的结果.

\section{2 分解反应 $\mathrm{R} 2$}

在对 $\mathrm{R} 1$ 的反应速率常数进行计算的基础上, 对 R2 的反应速率常数也进行了类似的计算. 在文献 [14]的实验条件范围内用非线性最小二乘法拟合, 得 到 R2 低压和高压极限的反应速率常数表达式分别 为

$$
\begin{aligned}
& k_{0}(2)=6.1 \times 10^{-3} \exp [-13011.9 / T] \\
& k_{\infty}(2)=4.0 \times 10^{16} \exp [-15463.1 / T]
\end{aligned}
$$

对比 R1 高压极限的反应速率常数指前因子, 发现 $\mathrm{R} 2$ 的与 $\mathrm{R} 1$ 的基本处于同一量级 $\left(10^{16} \mathrm{~s}^{-1}\right)$, 这说明 $\mathrm{PAN}$ 分解生成 $\mathrm{CH}_{3} \mathrm{C}(\mathrm{O}) \mathrm{O}+\mathrm{NO}_{3}$ 是一个经历疏松过 渡态的过程；但是 R2 拟合得到的阿仑尼乌斯活化 能是 $128.4 \mathrm{~kJ} \cdot \mathrm{mol}^{-1}$, 比 $\mathrm{Rl}$ 的 $112.5 \mathrm{~kJ} \cdot \mathrm{mol}^{-1}$ 大了 $15.9 \mathrm{~kJ} \cdot \mathrm{mol}^{-1}$, 直接原因是 $\mathrm{PAN}$ 中 $\mathrm{O}-\mathrm{O}$ 的 $132.6 \mathrm{~kJ} \cdot$ $\mathrm{mol}^{-1}$ 比 $\mathrm{O}-\mathrm{N}$ 的 $\mathrm{BDE}\left(118.8 \mathrm{~kJ} \cdot \mathrm{mol}^{-1}\right)$ 大 $(\mathrm{G} 3 \mathrm{MP} 2 \mathrm{~B} 3$ 的结果). 较大的断键能直接对应着较大反应能垒, 而较大的反应能垒直接导致了 $\mathrm{R} 2$ 的高压极限的反 应速率常数比 $\mathrm{R} 1$ 的低得多, 例如, 表 2 列出的结果 表明, $298 \mathrm{~K}$ 时的 R1 高压实验分解速率为 $10^{-4} \mathrm{~s}^{-1}$ 量级, 而同样温度 $8.0 \times 10^{4} \mathrm{~Pa}$ 气压下 $\mathrm{R} 2$ 的反应速率 常数只有 $8.9 \times 10^{-7} \mathrm{~s}^{-1}$. 也由于这个原因, $\mathrm{R} 2$ 的低压 


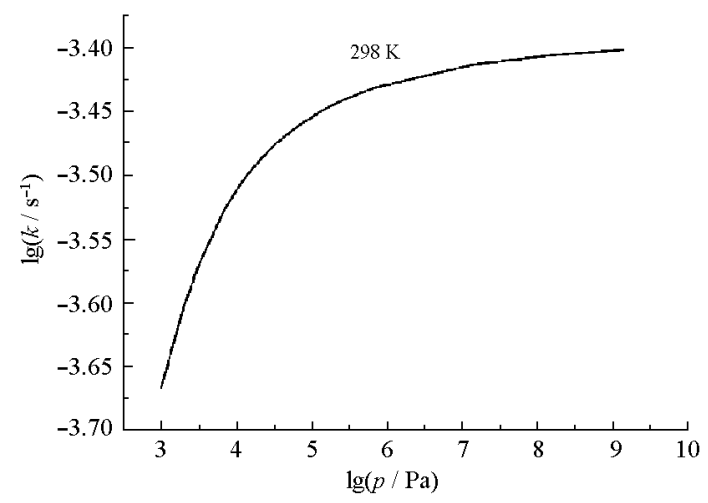

图 $3298 \mathrm{~K}$ 时 R1 分解反应速率常数随压强变化曲线

Fig.3 Pressure dependence of total rate constants for the reaction $\mathrm{R} 1$ at $298 \mathrm{~K}$

极限的反应速率常数也比 $\mathrm{R} 1$ 的低得多.

图 4 给出在 $1.0 \times 10^{3}$ 和 $8.0 \times 10^{4} \mathrm{~Pa}$ 压强下, 温度 为 $283 \sim 343 \mathrm{~K}$ 时 PAN 分解反应 $\mathrm{R} 2$ 的速率常数随 温度变化的曲线. 图 4 表明, 温度的变化对 $\mathrm{R} 2$ 的反 应速率常数影响同样很大, 并且随着温度升高, R2 的反应速率常数也逐渐增加. 对比图 2 和图 4 , 发现 $\mathrm{R} 2$ 分解反应的速率不管在低压和高压下都只有 $\mathrm{R} 1$ 的百分之一左右. 这说明 PAN 的热分解主要以 $\mathrm{R} 1$ 通道为主, $\mathrm{R} 2$ 为次要反应通道; 反应生成产物以 $\mathrm{PA}+$ $\mathrm{NO}_{2}$ 为主, $\mathrm{CH}_{3} \mathrm{C}(\mathrm{O}) \mathrm{O}+\mathrm{NO}_{3}$ 的生成占的比率很少. 同 时又由于生成物 $\mathrm{CH}_{3} \mathrm{C}(\mathrm{O}) \mathrm{O}$ 和 $\mathrm{NO}_{3}$ 的热不稳定性 ${ }^{[15]}$, $\mathrm{CH}_{3} \mathrm{C}(\mathrm{O}) \mathrm{O}$ 自由基比较容易分解为 $\mathrm{CH}_{3}$ 和 $\mathrm{CO}_{2}, \mathrm{NO}_{3}$ 具有很高的反应活性, 能够很快地和大气成分反应 而生成 $\mathrm{NO}_{2}\left(\mathrm{NO}_{3}+\mathrm{RH} / \mathrm{CO} / \mathrm{O}_{3} / \mathrm{H}_{2} \mathrm{O} \cdots \rightarrow \mathrm{NO}_{2}+\right.$ 其它产物), 这可能是长期以来 PAN 热分解实验 ${ }^{[9-12, ~ 14, ~ 16-20,241 ~}$ 都只 观察到 $\mathrm{NO}_{2}$ 产物的重要原因. 虽然 $\mathrm{R} 2$ 的反应速率

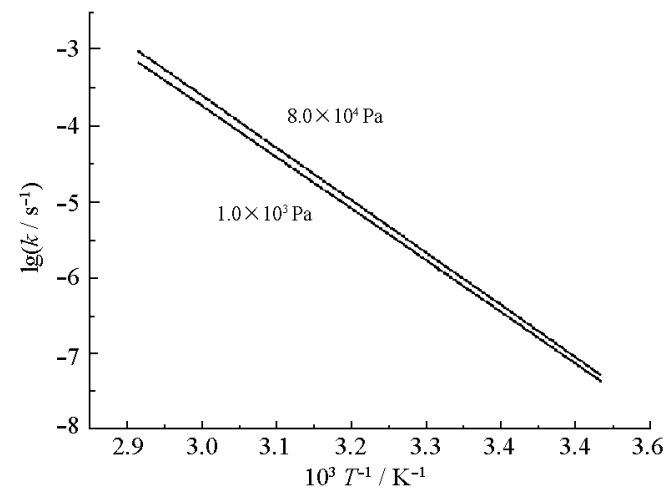

图 4 在 $1.0 \times 10^{3}$ 和 $8.0 \times 10^{4} \mathrm{~Pa}$ 压强下 $\mathrm{PAN}$ 分解反 应 $\mathbf{R} 2$ 的阿仑尼乌斯曲线

Fig.4 Arrhenius plots for the decomposition reaction $\mathrm{R} 2$ of $\mathrm{PAN}$ at the pressure of $1.0 \times 10^{3}$ and $8.0 \times 10^{4} \mathrm{~Pa}$

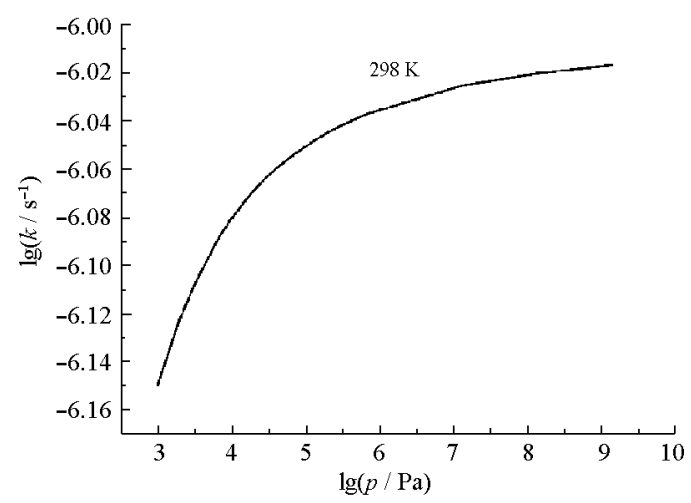

图 $5298 \mathrm{~K}$ 时 $\mathrm{R} 2$ 分解反应速率常数随压强变化曲线

Fig.5 Pressure dependence of total rate constants for the reaction $\mathrm{R} 2$ at $298 \mathrm{~K}$

常数相对反应 $\mathrm{R} 1$ 很小, 但最近 Ahsen 等人 ${ }^{[15]}$ 采用 光谱检测的方法证实 R2 的初级反应产物再次分解 所得的二级反应产物, 这说明 R2 反应也是可以发 生并且可以间接观测的. 图 5 给出 $298 \mathrm{~K}$ 时 $\mathrm{R} 2$ 的 分解反应速率常数随压强变化曲线.

从图 5 可看出, 这个温度下的高压反应速率常 数约为 $1.2 \times 10^{-6} \mathrm{~s}^{-1}$, 在 $1.0 \times 10^{4} \mathrm{~Pa}$ 左右反应速率常 数达到最大值的一半.

\section{4 结 论}

在 G3MP2B3 结构优化和能量计算的基础上, 采用 Troe 的 RRKM 计算方法, 用理论和疏松过渡 态模型计算了过氧硝酸乙酰酯热分解反应通道 R1 和 R2 分别随温度和压强变化的反应速率常数, 得 到低压和高压极限速率常数表达式(4) (7). 计算结 果表明, $\mathrm{R} 1$ 是主要的分解通道, R2 是次要通道, R2 的反应速率常数比 $\mathrm{R} 1$ 的小两个数量级.

\section{References}

1 Stephens, E. R. Adv. Environ. Sci. Technol., 1969, 1: 119

2 Roberts, J. M. Atmos. Environ. A, 1990, 24: 243

3 Stephens, E. R.; Hanst, P. L.; Doerr, R. C.; Scott, W. E. Ind. Chem. Eng., 1956, 48: 1498

4 Taylor, O. C. J. Air Pollu. Contr. Assoc., 1969, 19: 347

5 Stephan, S.; David, J. K.; Kerr, J. A. J. Phys. Chem. A, 1997, 101: 55

6 Singh, H. B.; Salas, L. J.; Viezee, W. Nature, 1986, 321: 588

7 Ridley, B. A.; Shetter, J. D.; Gandrud, B. W.; Salas, L. J.; Singh, H. B.; Carroll, M. A.; Hubler, G.; Albritton, D. L.; Hastie, D. R.; Schiff, H. I.; Mackay, G. I.; Karechi, D. R.; Davis, D. D.; 
Bradshaw, J. D.; Rodgers, M. O.; Sandholm, S. T.; Torres, A. L.; Condon, E. P.; Gregory, G. L.; Beck, S. M. J. Geophys. Res. (D7), 1990, 95: 10179

8 Miller, C. E.; Lynton, J. I.; Keevil, D. M. J. Phys. Chem. A, 1999, 103: 11451

9 Roberts, J. M.; Bertman, S. B. Int. J. Chem. Kinet., 1992, 24: 297

10 Tuazon, E. C.; Carter, W. P. L.; Atkinson, R. J. Phys. Chem., 1991, 95: 2434

11 Grosjean, D.; Grosjean, E.; Williams, II E. L. J. Air Waste Manag. Assoc., 1994, 44: 391

12 Cox, R. A.; Roffey, M. J. Environ. Sci. Technol., 1977, 11: 900

13 Hendry, D. G.; Kenley, R. A. J. Am. Chem. Soc., 1977, 99: 3198

14 Bridier, I.; Caralp, F.; Loirat, H.; Lesclaux, R.; Veyret, B.; Becker, K. H.; Reimer, A.; Zabel, F. J. Phys. Chem., 1991, 95: 3594

15 Ahsen, S. V.; Willner, H.; Francisco, J. S. J. Chem. Phys., 2004, 121: 2048

16 Nieboer, H.; Duyzer, J. H. Photochemical smog formation in the Netherlands. Dutch: Guicherit R, TNO Research Institute for Environmental Hygiene, 1978:89

17 Schurath, U.; Wipprecht, V. Proceedings of the First European Symposim on Physio-Chemical Behavior of Atmospheric Pollutants, Version B. Ispra, Italy: Ott, H.(Ed.), Commission of the European Communities, 1979

18 Orlando, J. J.; Tyndall, G. S.; Calvert, J. G. Atmos. Environ. A, 1992, 26: 3111

19 Sehested, J.; Christensen, L. K.; Mogelberg, T.; Nielsen, O. J.; Wallington, T. J.; Guschin, A.; Orlando, J. J.; Tyndall, G. S. J. Phys. Chem. A, 1998, 102: 1779

20 Kirchner, F.; Mayer-Figge, A.; Zabel, F.; Becker, K. H. Int. J. Chem. Kinet., 1999, 31: 127

21 Mhin, B. J.; Chang, W. Y. J. Phys. Chem. A, 2000, 104: 2613

22 Domalski, E. S. Environ. Sci. Technol., 1971, 5: 443

23 Jursic, B. S. THEOCHEM, 1996, 370: 65
24 Zabel, F. Z. Phys. Chem., 1995, 188: 119

25 Harwood, M. H.; Roberts, J. M.; Frost, G. J.; Ravishankara, A. R.; Burkholder, J. B. J. Phys. Chem. A, 2003, 107: 1148

26 Mazely, T. L.; Friedl, R. R.; Sander, S. P. J. Phys. Chem., 1995, 99: 8162

27 Mazely, T. L.; Friedl, R. R.; Sander, S. P. J. Phys. Chem. A, 1997, 101: 7090

28 Kleindienst, T. E. Res. Chem. Intermed., 1994, 20: 335, and references cited therein.

29 Kasting, J. F.; Singh, H. B. J. Geophys. Res. (DI2), 1986, 91: 13239

30 Gardiner, Jr. W. C.; Troe, J. Combustion chemistry. New York: Springer-Verlag, 1984

31 Troe, J. Ber. Bunsenges. Phys. Chem., 1983, 87: 161

32 Gilbert, R. G.; Luther, K.; Troe, J. Ber. Bunsenges. Phys. Chem., 1983, 87: 169

33 Wei, W. M.; Tan, W.; Zheng, R. H.; He, T. J.; Chen, D. M.; Liu, F. C. Chem. Phys., 2005, 312: 241

34 Becke, A. D. J. Chem. Phys., 1993, 98: 5648

35 Lee, C.; Yang, W.; Parr, R. G. Phys. Rev. B, 1988, 37: 785

36 Baboul, A. G.; Curtiss, L. A.; Redfern, P. C.; Raghavachari, K. J. Chem. Phys., 1999, 110: 7650

37 Frisch, M. J.; Trucks, G. W.; Schlegel, H. B.; et al. Gaussian 03. Revision B.03. Pittsburgh, PA: Gaussian, Inc., 2003

38 Stiel, L. I.; Thodos, G. J. Chem. Eng. Data, 1962, 7: 234

39 Bruckmann, P. W.; Willner, H. Emiron. Sci. Technol., 1983, 17: 352

40 Reid, R. C.; Sherwood, T. K. The properties of gases and liquids. 2nd ed. New York: McGraw-Hill, 1996

41 Forst, W. Theory of unimolecular reaction. New York: Academic Press, 1973

42 Caralp, F.; Lesclaux, R.; Rayez, M. T.; Rayez, J. C.; Forst, W. J. Chem. Soc. Faraday Trans. 2, 1988, 84: 569 